\title{
Simple Surface Functionalization Strategy for Immunosensing Detection of Aflatoxin B1
}

\author{
Mihaela Badea ${ }^{1}$, Laura Floroian $^{2, *}$, Patrizia Restani $^{3}$, Marius Moga $^{4}$ \\ ${ }^{1}$ Fundamental, Prophylactic and Clinical Specialties Department, Transilvania University of Brasov, \\ 29 Eroilor Blvd, 500036, Brasov, Romania \\ ${ }^{2}$ Automation and Information Technology Department, Transilvania University of Brasov, 29 Eroilor \\ Blvd, 500036, Brasov, Romania \\ ${ }^{3}$ Pharmacological and Biomolecular Sciences Department, Università degli Studi di Milano, Via Festa \\ del Perdono, 7, 20122, Milano, Italy \\ ${ }^{4}$ Department of Medical and Surgical Specialties, Transilvania University of Brasov, 29 Eroilor Blvd, \\ 500036, Brasov, Romania \\ *E-mail: lauraf@unitbv.ro
}

doi: $10.20964 / 2016.08 .21$

Received: 24 April 2016 / Accepted: 28 April 2016 / Published: 7 July 2016

This paper describes the strategy of functionalization of gold electrodes surfaces in aim to obtain a new impedimetric immunosensor for the detection of aflatoxin B1, a common toxic food contaminant. The immunosensor elaboration is based on immobilization of anti-aflatoxin antibody on gold electrodes modified with a cross-linked film of bovine serum albumin, by a four-step protocol. The immunosensor is based on a simple design and requires small volumes of toxic aflatoxin solution. All the steps of the immunosensor elaboration and the immunochemical reaction between aflatoxin and antibody were followed using atomic force microscopy (AFM) and electrochemical impedance spectroscopy (EIS). The resistance to charge transfer (Rct) was the most sensitive parameter to changes induced to the interfacial properties of the immunosensor by the incubation with aflatoxin and varied linearly with aflatoxin concentration in the range $1-20 \mathrm{ng} / \mathrm{mL}$. The immunosensor was applied for the detection of aflatoxin in spiked plant extracts with good recovery factors.

Keywords: surface functionalization; aflatoxin B1 detection; impedimetric immunosensor; electrochemical impedance spectroscopy.

\section{FULLTEXT}

(C) 2016 The Authors. Published by ESG (www.electrochemsci.org). This article is an open access article distributed under the terms and conditions of the Creative Commons Attribution license (http://creativecommons.org/licenses/by/4.0/). 